\title{
Inserción del eje transversal de Gestión Integral de Riesgo y Cambio Climático con enfoque de Gobernabilidad, en la asignatura de Gestión de la Energía, durante el primer semestre del 2013
}

\author{
Incorporation of the cross curricular themes of Integral Risk Management \\ and Climate Change with Governability approach, on the subject of Energy \\ Management, during the first semester of 2013
}

\author{
Edwin Antonio Reyes Aguilera ${ }^{1}$ \\ Rubén Antonio Dormus Centeno² \\ Porfirio José Pérez Guevara ${ }^{3}$
}

1 UNAN-Nanagua FAREM-Estelí. Correo Electrónico: edwinra11@yahoo.es

2 UNAN-Nanagua FAREM-Estelí. Correo Electrónico: rubendorcen@gmail.com

3 UNAN-Nanagua FAREM-Estelí. Correo Electrónico: pp_guevaraj@yahoo.es

\section{RESUMEN}

El proceso de inserción del eje transversal de Gestión de Riesgo y Cambio Climático, en la asignatura de Gestión de la Energía, de la carrera de Ingeniería en Energías Renovables durante el primer semestre del 2013, se realizó la asignatura Gestión de la Energía, con docentes y estudiantes de $\mathrm{V}$ año del turno vespertino, específicamente en el tema de: Centrales Eléctricas, y su relación con el cambio climático y el enfoque de gobernabilidad. Esta ha sido una investigación acción, inicio con entrevistas a los docentes, identificando la situación problema y se concluye que es necesario hacer una bue $\urcorner$ na planificación utilizando estrategias metodológicas innovadoras que permita un proceso de reflexión crítica y que consideren el análisis de la problemática del contexto para generar aprendizajes significativos. Los participantes en este proceso fueron 10 estudiantes varones.

Palabras Claves: Gestión Integral del Riesgo, gestión de la energía, cambio climático, gobernancia, incorporación de la perspectiva.

\section{ABSTRACT}

The process of the incorporation of the cross curricular themes of Integral Risk Management and Climate Change with Governability approach, on the subject of Energy Management, during the first semester of 2013, the ssubject of Energy Management was conducted with teachers and students of Fifth grade of the vespertine shift, specifically on the topic of: Power plants, and its relation with the climate change and the Governability approach. This is an action research, that began with the interviews to the teachers, identifying the problem, and concludes that it is necessary to do a good planning by using innovative methodological strategies that permit a process of critical reflection and consider analyzing the problem, generating meaningful learning. During this process participated 10 male students.

Keywords: Integral Risk Management, energy management, climate change, governance, mainstreaming. 


\section{INTRODUCCION}

En este marco es imperiosa la necesidad que los países adopten Estrategias Nacionales, desde el más alto nivel, para abordar la problemática de manera holística, integral e integrada con una cabal comprensión de la complejidad del fenómeno y sea desde las instancias de Planificación del Estado que se emanen los lineamientos y políticas en los diferentes ámbitos.

Es necesario que la educación, como sistema y como función, dinamice la coordinación y vinculación activa de los organismos y entidades públicas y privadas de todos los sectores, y su directa relación con la sociedad civil en los procesos participativos, aportando la formación y concienciación necesarias para la GRCC, del mismo modo, potenciar el reconocimiento de los centros educativos como actores destacados en el marco de esta problemática, donde la estructura del proceso sea establecer la coherencia entre la proyección y formación de los docentes. La educación como polo de desarrollo constituye una base fundamental para comprender la dimensión social de la GRCC y a partir de ahí promover un sistema de conocimientos y cultura ciudadana frente al riesgo.

La investigación desarrollada se realizó en la Facultad Regional Multidisciplinaria de Estelí, en la asignatura Gestión de la Energía, con docentes y estudiantes de $\mathrm{V}$ año, de la carrera de ingeniería en Energías Renovables en el turno vespertino, específicamente en el tema de: Centrales Eléctricas, donde se abordó la relación de estas con el cambio climático y el enfoque de gobernabilidad.

Durante el proceso de deliberación sobre la idea de la investigación, se realizó un proceso de reflexión a partir de la siguiente pregunta ¿Cuál es el conocimiento necesario para poder proceder a realizar una transversalizacion? Luego de llegar a un consenso planteamos las siguientes preguntas:
¿Cuál es el procedimiento para llevar a cabo una transverzalización? ¿Qué nos limita desde el punto de vista metodológico? ¿Cuál es nuestro nivel de conocimiento en la temática de Gestión integral del riesgo y cambio Climático? ¿Cuál es la importancia de realizar esta transverzalización?

Con el propósito de la incorporación de la ges $\neg$ tión de riesgos y cambio climático con enfoque de gobernabilidad con el fin de alcanzar cambios de actitudes en los estudiantes, a través del análisis y reflexion, que les permita Valorar los aspectos positivos y negativos producidos por las centrales eléctricas, durante el proceso de construcción y generación de la energía eléctrica así mismo identificando los daños que causan al medio ambiente y las leyes que regulan su funcionamiento. Llegando a un consenso, luego de una reflexión más detallada, elaboramos la propuesta de investigación, ¿Cómo insertar el eje de Gestión Integral del Riesgo y Cambio Climático, con enfoque de gobernabilidad en la asignatura de Gestión de la Energía?,

El enfoque metodológico utilizado fue de una Investiga cción Acción, se parte de la reflexión crítica sobre nuestra propia práctica para luego experimentar nuevas estrategias, evaluar y llegar a una práctica mejorada.

\section{ETAPA DIAGNÓSTICA}

Para desarrollar esta investigación se describe el procedimiento metodológico utilizado, considerando que ésta es importante sobre todo, para mejorar nuestra práctica educativa. En el proceso de esta investigación, se realizó una diagnosis sobre el conocimiento y la inserción del eje transversal en gestión de riesgo, cambio climático y gobernabilidad, de los docentes de la carrera de Ingeniería en energías renovables en la FAREM-Estelí, esto permitió reflexionar sobre las siguientes preguntas que nos guiaron en el proceso. 
1. ¿Cuál es nuestro nivel de preparación para insertar ejes transversales en la carrera de ingeniería en energías renovables?

2. ¿Cuáles son los problemas metodológicos que tenemos para realizar una planificación con el propósito de insertar ejes transversales?

3. ¿Qué tanto están conscientes y apropiados los maestros para insertar ejes transversales en las diferentes asignaturas de energías renovables?

4. ¿Cuáles son las estrategias que conocemos y podemos aplicar para la inserción de ejes transversales?

5. ¿Cuál es el conocimiento inicial de los estudiantes sobre estos temas?

6. ¿Cuál es la motivación del estudiante por ampliar sus conocimientos sobre la gestión de riesgo y cambio climático?

Inicialmente se entrevistó a 5 docentes que imparten asignaturas en la carrera de ingeniería en energías renovables, en relación a la inserción de ejes transversales específicamente en los temas relacionados a gestión de riesgo, cambio climático y gobernabilidad.

De las entrevistas a los docentes, podemos asegurar que 2 han insertado ejes transversales porque han estudiado este mismo diplomado y ha sido parte de su formación y se sienten motivados y concienciados en estos temas y consideran importante transferir este conocimiento a los dicentes. Esto nos permitió conocer algunas estrategias metodológicas para implementarlas en el desarrollo de la inserción del eje transversal y generar aprendizajes significativos.

Según los docentes que insertan el eje, inicialmente revisan su contenido temático donde les facilite la transversalización para de esta manera elaborar el diseño metodológico de la sesión.

En las asignaturas de la especialidad se identifican con facilidad las temáticas donde se pueden insertar los ejes transversales y es más fácil su aplicación, dada la naturaleza de la carrera, sin embargo existen otras asignaturas donde sea hace más difícil transversalizar, ya que el contenido no poseen mucha relación con las temática de gestión de riesgo, cambio climático y gobernabilidad.

Entre las estrategias metodológicas que han aplicado los docentes que han insertado el eje transversal en gestión de riesgo y cambio climático, tenemos: panel, giras de campo, video foros, conferencias, entre otros, en actividad conjunta en el proceso, aplicando diferentes técnicas didácticas.

En referencia a los tres docentes que no han insertado los ejes transversales, respondieron que no lo han hecho por desconocimiento al tema y no consideran importante esta actividad y consideran que para abordar esta temática es suficiente con enviar al dicente a investigar.

\section{ETAPA DE ACCIÓN}

\section{Hipótesis de acción}

Cuanto mayor sea el nivel de apropiación y concienciación de los docentes en la inserción de ejes transversales en gestión de riesgo, mayor será el nivel de comprensión de los dicentes en energías renovables en estos temas.

\section{Principales acciones realizadas}

Inicialmente se realizó la revisión del plan didáctico para identificar dentro del contenido la parte en que se podría insertar la temática de gestión de riesgo, cambio climático y gobernabilidad. Luego de la revisión del contenido y de una reflexión sobre el tema a transversalizar, se procede a escoger la unidad número 8 , que contiene la temática de las centrales eléctricas y en la que se consideró, nos permitía realizar la inserción del eje. Seguidamente se procede a adecuar los objetivos de la unidad de tal manera que nos permita el cumplimiento del objetivo durante 
el proceso de la inserción del eje transversal. Luego se procedió a elaborar el plan de clase utilizando una metodología tipo taller.

La sesión se inició con una conferencia sobre las diferentes centrales eléctricas, en el cual durante el desarrollo de la temática, se insertaba el eje de gestión de riesgo, cambio climático y gobernabilidad; la clase se realizó de forma interactiva, lo que permitió darnos cuenta del nivel de conocimiento de los estudiantes.

La actividad 2, consistió en presentar un video sobre el cambio climático, sus causas y consecuencias, entre las que se identificaban la emanación de gases efecto invernadero (GEI), derivados de la quema de combustibles fósiles en las centrales eléctricas; una vez finalizado el video se procede a su discusión para lo cual se orientaron preguntas reflexivas sobre el mismo.

La actividad tres, consistió en formar dos grupos de 3 y un grupo 4 estudiantes, cada grupo seleccionó una temática: Centrales hidroeléctricas, Centrales termoeléctricas y centrales eólicas, en la cual identificaron, amenazas y vulnerabilidades en las áreas donde se establecen las centrales eléctricas, para esto se les facilitó material para desarrollar el trabajo.

Luego cada grupo presentó en plenario el trabajo, apoyados de papelógrafos, en este proceso se aclaraban ciertas dudas que surgían relacionados al cambio climático. Al finalizar, se hizo una conclusión de todo el proceso en el aula de clase, de igual manera se brindó espacio a los estudiantes para que dieran su valoración a la actividad realizada y la metodología empleada.

\section{ETAPA DE EVALUACIÓN}

\section{Logros}

- Diseño y ejecución de un plan de acción, que nos brindó gran satisfacción, sobre todo con la obtención de logros positivos en los estudiantes, por la motivación y disposición de estos en el desarrollo de la sesión.

- Estudiantes manifestaban el interés por introducir esta temática en dos trabajos de curso que estaban desarrollando, lo cual se logró constatar en los trabajos entregados a los docentes, de los que uno de los facilitadores de esta sesión, había orientado a los estudiantes.

- El mayor logro lo representa, la inserción de la temática en la asignatura y de esta manera lograr mayor conciencia y sensibilización, así como el cambio de actitud manifiesta de los estudiantes

- Identificar que esta temática puede adecuarse a los contenidos de otras asignaturas, de las cuales somos facilitadores.

- Motivar y que los alumnos se integraran a las diferentes actividades que se realizaron en la sesión, esto generó un proceso de discusión crítica, constructivo e interactivo entre los integrantes y los facilitadores.

- Aplicación de estrategias metodológicas innovadoras que facilitan el aprendizaje significativo en los estudiantes.

\section{Dificultades y elementos obstaculizadores}

- Falta de experiencia en inserción de ejes transversales de cualquier temática, debido a que los miembros del equipo facilitador nunca habían abordado este tema a manera de inserción.

- Un elemento obstaculizador fue no disponer de tiempo necesario para la elaboración del plan de acción, debido a las múltiples actividades que cada miembro del equipo facilitador tiene dentro de la universidad.

- Desconocimiento de algunas estrategias metodológicas adecuadas por parte del equipo investigador. 


\section{CONCLUSIONES}

Las actividades desarrolladas en el grupo de quinto año de ingeniería en energías renovables, con los que se estableció el plan de acción, pusieron en evidencia elementos positivos alrededor de la transversalización del tema abordado. Se estableció con los estudiantes actividades que favorecieron la construcción de conocimiento a través de la discusión en equipo y en plenario.

Mientras los estudiantes observaban el video era impresionante ver la manera de como se asombraban viendo el impacto que tiene el cambio climático al medioambiente y los impactos en la población, eso demuestra la sensibilidad ante estas situaciones. Esto facilitó el espacio para compartir, reflexionar y poner en común ideas.

Luego de realizar un análisis exhaustivo de las diferentes actividades ejecutadas, desde el punto de vista del propósito de la investigación, se puede decir categóricamente, que el observar situaciones reales y contextualizadas; permite sensibilizar y concientizar a las personas logrando de esta forma, cambios de actitudes que le serán de utilidad en su vida personal y profesional.

Este tipo de sesión con estrategia innovadora permitió en los estudiantes la integración y participación en actividades y el deseo de apoyar en la facilitación de estos temas en otras asignaturas.

Durante el proceso de la investigación reconocimos la factibilidad de identificar las asignaturas donde podemos insertar los ejes temáticos y planificar nuestras clases en función de ejes transversales.

\section{BIBLIOGRAFIA}

Milán, J. A. (2012). Dossier Introducción al Cambio Climático. Estelí.

Morales, V., \& Trejos, R. (2008). ABC Cambio Climático, Nicaragua (Primera ed.). Managua.

Valenzuela, Y., Alonso, S., Picado, A., \& Herrera, R. (2012). "Inserción de Gestión Integral de Riesgo y Cambio Climático con enfoque de Género en las asignaturas de Estructura Económica de Nicaragua, Introducción a la Biología, Principios de Administración y Prácticas de Profesionalización durante el primer semestre. Estelí. 